\title{
Quantitative shearography: error reduction by using more than three measurement channels
}

\author{
Tom O. H. Charrett, Daniel Francis, and Ralph P. Tatam* \\ Department of Engineering Photonics, School of Engineering, Cranfield University, \\ Cranfield, Bedford, MK43 OAL, UK \\ ${ }^{*}$ Corresponding author: r.p.tatam@ cranfield.ac.uk
}

Received 13 October 2010; revised 11 November 2010; accepted 14 November 2010; posted 23 November 2010 (Doc. ID 136560); published 5 January 2011

\begin{abstract}
Shearography is a noncontact optical technique used to measure surface displacement derivatives. Full surface strain characterization can be achieved using shearography configurations employing at least three measurement channels. Each measurement channel is sensitive to a single displacement gradient component defined by its sensitivity vector. A matrix transformation is then required to convert the measured components to the orthogonal displacement gradients required for quantitative strain measurement. This transformation, conventionally performed using three measurement channels, amplifies any errors present in the measurement. This paper investigates the use of additional measurement channels using the results of a computer model and an experimental shearography system. Results are presented showing that the addition of a fourth channel can reduce the errors in the computed orthogonal components by up to $33 \%$ and that, by using 10 channels, reductions of around $45 \%$ should be possible. (C) 2011 Optical Society of America
\end{abstract}

OCIS codes: $100.3175,110.3175,110.6150,120.0120,120.6160,120.6165$.

\section{Introduction}

Surface strain measurement is important in the characterization of the reliability, strength, and lifetime of components in many branches of engineering. Examples include the characterization of gas turbine blades, automobile parts, and composite materials. Surface strain is often measured using electrical strain gauges, which are adhered to the surface of the component of interest. A limitation with strain gauges is that the measurement is only made at the location at which it is attached to the surface with a spatial resolution determined by the dimensions of the gauge. Also, the surface of the component to which the strain gauge is to be bonded may require significant preparation before the gauge will adhere to it sufficiently to ensure high fidelity of strain transfer from the part to the gauge. Certain optical measurement techniques, such as speckle metrology, can provide a solution to these problems due to their

0003-6935/11/020134-13\$15.00/0

(C) 2011 Optical Society of America noncontact nature and their ability to provide a measurement across an extended field of view.

Speckle shearing interferometry [1-3], commonly known as shearography, is an interferometric speckle technique that is sensitive to changes in the displacement gradient of surfaces under an applied load. The technique is often used in the automotive and aerospace industries for qualitative nondestructive analysis, for example, in the detection of disbonds and defects in composite materials [4].

\section{A. Single-Channel Shearography}

The optical arrangement for a single-channel shearography system that is predominantly sensitive to an out-of-plane displacement gradient is shown in Fig. 1. The arrangement shown here is based on a shearing Michelson interferometer. Coherent light from the laser is expanded to illuminate a region of interest on the target's surface. Light scattered from the target is imaged onto the charge-coupled device (CCD) camera via an image shearing device, producing a speckle pattern on the CCD array. The shearing device is commonly a Michelson interferometer with 


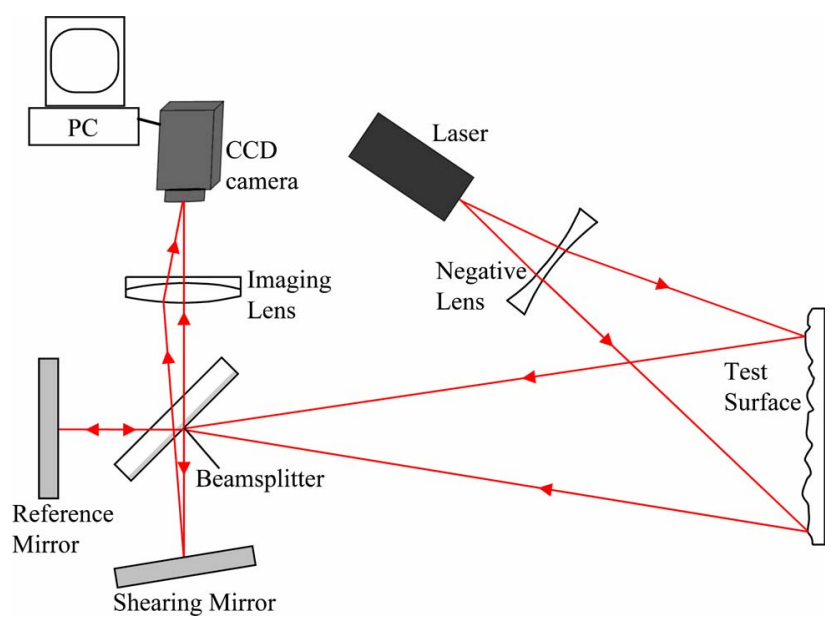

Fig. 1. (Color online) Typical single-channel shearography arrangement based on a Michelson interferometer.

an off-axis tilt of one of the mirrors relative to the other, as shown in Fig. 1. The tilt between the mirrors results in the coherent combination of identical, but displaced, (sheared) images, producing an interferometric speckle pattern at the CCD array that is sensitive to relative changes in the phase of light scattered from neighboring points on the illuminated surface. When the object is deformed, a change in the intensity of the individual speckles is observed. When making a measurement, interferometric speckle patterns are recorded before and after deformation of the object. The two images, known as the reference frame (before deformation) and the signal frame (after deformation) are correlated by digital subtraction of the intensities recorded at each pixel in the CCD array. The resulting image consists of dark and light bands where the two speckle patterns are in-phase and anti-phase, respectively, and is known as a correlation fringe pattern. The fringes represent a locus of points with the same magnitude of displacement gradient. Phase analysis techniques can be used to recover the phase distribution across the object surface, which is, in general, a combination of the in-plane and out-ofplane components of the deformation gradients on the object surface. This is often achieved using the technique known as temporal phase stepping [5], which involves the recording of a series of images with a relative, and usually known, phase shift between them. The images are then combined using a phasestepping algorithm to yield the phase. These phase values are wrapped between $-\pi$ and $+\pi$. To obtain a continuous phase measurement, the $2 \pi$ discontinuities need to be removed using a process known as phase unwrapping $[\underline{3}, \underline{6}]$.

\section{B. Multicomponent Shearography}

A single shearography measurement channel is sensitive to the gradient of a component of the surface displacement. The direction of this component is determined by the sensitivity vector of the shearography instrument, as shown in Fig. 2. The sensitivity

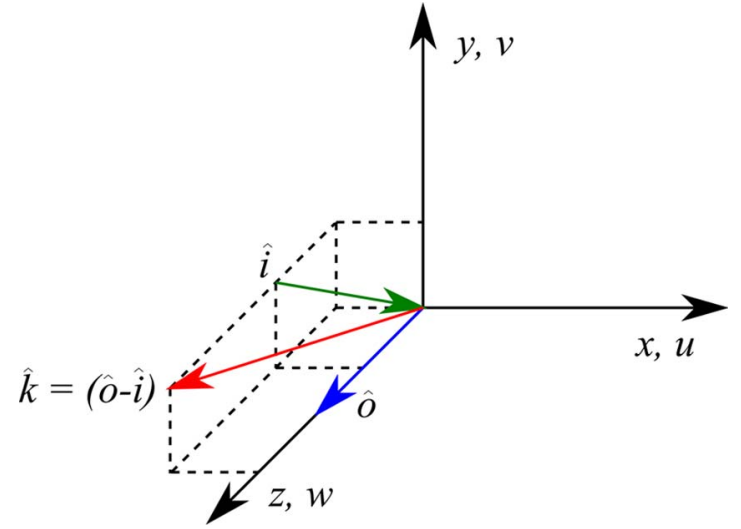

Fig. 2. (Color online) Relationship between the Cartesian $x, y$, and $z$ directions and the displacement components $u, v$, and $w$. The component of displacement gradient that a shearography instrument measures is determined by the sensitivity vector, $\hat{k}$, which is the bisector of the observation, $\hat{o}$, and illumination, $\hat{i}$, unit vectors.

vector, $\hat{k}$, is defined as the bisector of the observation vector, $\hat{o}$, and illumination vector $\hat{i}$ :

$$
\hat{k}=\hat{o}-\hat{i} \text {. }
$$

The phase distributions from a single shearography channel with the shear in the $x$ direction (horizontally) and $y$ direction (vertically), respectively, are given by

$$
\begin{aligned}
& \Delta \phi_{x}=\frac{2 \pi}{\lambda}\left[k_{x} \frac{\partial u}{\partial x}+k_{y} \frac{\partial v}{\partial x}+k_{z} \frac{\partial w}{\partial x}\right] d x, \\
& \Delta \phi_{y}=\frac{2 \pi}{\lambda}\left[k_{x} \frac{\partial u}{\partial y}+k_{y} \frac{\partial v}{\partial y}+k_{z} \frac{\partial w}{\partial y}\right] d y,
\end{aligned}
$$

where $u, v$ are the in-plane and $w$ is the out-of-plane components of the displacement vector, $\hat{U}=(u, v, w)$. The relationship between the displacement vector components and the $x, y$, and $z$ directions is illustrated in Fig. 2.

In general, a shearography phase measurement will contain contributions from all three orthogonal displacement gradient components: $\frac{\partial u}{\partial x}, \frac{\partial v}{\partial x}$, and $\frac{\partial w}{\partial x}$; or $\frac{\partial u}{\partial y}, \frac{\partial v}{\partial y}$, and $\frac{\partial w}{\partial y}$. To retrieve these components, at least three separate phase measurements are required, each with a different sensitivity vector. This can be achieved in a practical system either by using multiple illumination directions and a single observation direction and performing the measurements sequentially [7-9], or by using multiple observation directions and a single illumination direction and performing the measurements in parallel. This latter method can be more easily applied using imaging fiber bundles [10,11], where each view is ported to a quadrant of a single CCD camera. 
For multiple channels, this can be written as the matrix equations

$$
\begin{aligned}
& {\left[\begin{array}{c}
\Delta \phi_{x 1} \\
\Delta \phi_{x 2} \\
\Delta \phi_{x 3} \\
\vdots
\end{array}\right]=\frac{2 \pi}{\lambda}\left[\begin{array}{ccc}
k_{x 1} & k_{y 1} & k_{z 1} \\
k_{x 2} & k_{y 2} & k_{z 2} \\
k_{x 3} & k_{y 3} & k_{z 3} \\
\vdots & \vdots & \vdots
\end{array}\right]\left[\begin{array}{c}
\partial u / \partial x \\
\partial v / \partial x \\
\partial w / \partial x
\end{array}\right] d x,} \\
& {\left[\begin{array}{c}
\Delta \phi_{y 1} \\
\Delta \phi_{y 2} \\
\Delta \phi_{y 3} \\
\vdots
\end{array}\right]=\frac{2 \pi}{\lambda}\left[\begin{array}{ccc}
k_{x 1} & k_{y 1} & k_{z 1} \\
k_{x 2} & k_{y 2} & k_{z 2} \\
k_{x 3} & k_{y 3} & k_{z 3} \\
\vdots & \vdots & \vdots
\end{array}\right]\left[\begin{array}{c}
\partial u / \partial y \\
\partial v / \partial y \\
\partial w / \partial y
\end{array}\right] d y .}
\end{aligned}
$$

Here, $k_{x}, k_{y}$, and $k_{z}$ are the $x, y$, and $z$ components of the sensitivity vector and the subscripts refer to the particular measurement channel. The orthogonal displacement gradient components are then calculated using a matrix transformation.

This paper investigates the use of additional measurement channels in shearography and, in particular, their influence on the transformation to the orthogonal strain components. In previous work [7-11], the number of channels used in this transformation has been limited to three, allowing Eqs. (4) and (5) to be solved directly. However, the transformation results in the amplification of errors present in the data due to the arrangement of the sensitivity vectors.

A fourth measurement channel can be easily added to a shearography system, by either the addition of a fourth illumination direction, an additional viewing direction, or additional shearing interferometer and camera. Groves et al. [9] described a system with four measurement channels. Here the fourth channel allows the surface strain to be fully characterized when one of the channels has poor correlation fringe quality, or is obstructed as a result of the object shape. In this situation, the fourth channel can be used to replace the channel that has poor quality data in the transformation. Similarly, systems using coherent imaging fiber bundles $[10,11]$ have four channels available. However, in these systems, only three channels are employed in the calculation of the orthogonal strain components and the remaining data is discarded. Previous work in the field of planar Doppler velocimetry $[12,13]$, using a similar coordinate transformation, has shown that the use of a fourth channel in the calculation can significantly improve the results and lead to a reduction in the errors propagated to the final calculated orthogonal velocity components. For geometries similar to those typically found in shearography systems, these reductions can be as much as $25 \%$ of the error [13].

A derivation of the equations used to calculate the orthogonal components using four or more channels is given in the next section of this paper, followed by a description of a four-channel experimental shearography system and a computer model for an $n$-channel shearography system. A comparison between results calculated using three or four channels is then presented using results of the computer model and experimental data for a series of test displacement fields. Finally the benefits of using more than four channels are assessed using the computer model.

\section{Calculation of the Orthogonal Strain Components Using Four (or More) Channels}

As Eqs. (4) and (ㄷ) are similar, the derivation presented here is in terms of the shear, $d s$, which can be in either the horizontal $(d s=d x)$ or vertical direction $(d s=d y)$. The result is given in general terms for $n$ measurement channels, where $n \geq 3$. Equations ( $\underline{4})$ and (5) can be written as

$$
\Phi=\left(\frac{2 \pi d s}{\lambda}\right) J M,
$$

where $\Phi$ is a column vector containing the measured phases, and $J$ is the matrix constructed from the sensitivity vector components with each row containing $k_{x}, k_{y}$, and $k_{z}$ for a single shearography channel. $M$ is a column vector containing the displacement gradient components and $d s$ is the magnitude of the shear. For three channels, this can be solved by matrix inversion:

$$
M=\left(\frac{\lambda}{2 \pi d s}\right) J^{-1} \Phi .
$$

However, if more than three measurements are made, Eqs. (4) and (5) become overdetermined and can be solved using the general least-squares method for linear equations [14]:

$$
M=\left(\frac{\lambda}{2 \pi d s}\right)\left(J^{T} W J\right)^{-1} J^{T} W \Phi .
$$

Here the superscript $T$ indicates the transpose of a matrix and $W$ is a weighting matrix, which allows each channel to ranked, with larger values, $W_{n}$, indicating greater confidence:

$$
W=\left[\begin{array}{cccc}
W_{1} & 0 & 0 & \ldots \\
0 & W_{2} & 0 & \ldots \\
0 & 0 & W_{3} & \ldots \\
\vdots & \vdots & \vdots & W_{n}
\end{array}\right]
$$

Equation (8) can be expanded into general solutions for $\frac{\partial u}{\partial s}, \frac{\partial v}{\partial s}$, and $\frac{\partial w}{\partial x}$ using $n$ channels:

$$
\frac{\partial u}{\partial s}=\left(\frac{\lambda}{2 \pi d s}\right) \frac{[g(d f-e e)+h(c e-b f)+j(b e-c d)]}{[a(d f-e e)-b(b f-c e)+c(b e-d c)]},
$$

$$
\frac{\partial v}{\partial s}=\left(\frac{\lambda}{2 \pi d s}\right) \frac{[g(c e-b f)+h(a f-c c)+j(b c-a e)]}{[a(d f-e e)-b(b f-c e)+c(b e-d c)]},
$$




$$
\frac{\partial w}{\partial s}=\left(\frac{\lambda}{2 \pi d s}\right) \frac{[g(b e-c d)+h(b c-a e)+j(a d-b b)]}{[a(d f-e e)-b(b f-c e)+c(b e-d c)]}
$$

where

$$
\begin{aligned}
a & =\sum_{i}^{n} k_{x i}{ }^{2} W_{i}, \quad b=\sum_{i}^{n} k_{x i} k_{y i} W_{i}, \\
c & =\sum_{i}^{n} k_{x i} k_{z i} W_{i}, \quad d=\sum_{i}^{n} k_{y i}{ }^{2} W_{i}, \\
e & =\sum_{i}^{n} k_{y i} k_{z i} W_{i}, \quad f=\sum_{i}^{n} k_{z i}{ }^{2} W_{i}, \\
g & =\sum_{i}^{n} k_{x i} \Delta \phi_{i} W_{i}, \quad h=\sum_{i}^{n} k_{y i} \Delta \phi_{i} W_{i}, \\
j & =\sum_{i}^{n} k_{z i} \Delta \phi_{i} W_{i} .
\end{aligned}
$$

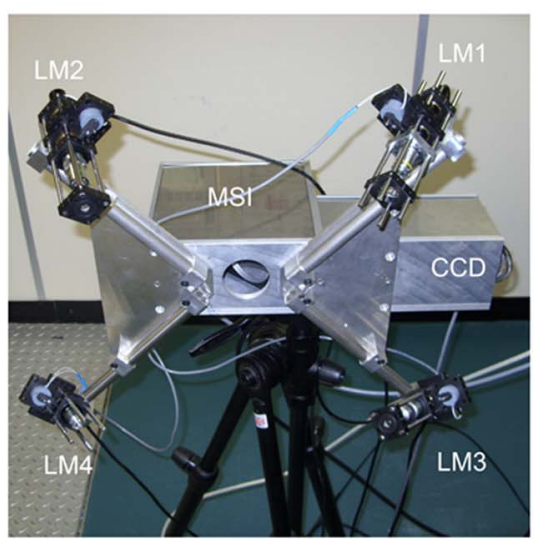

PHOTOGRAPH

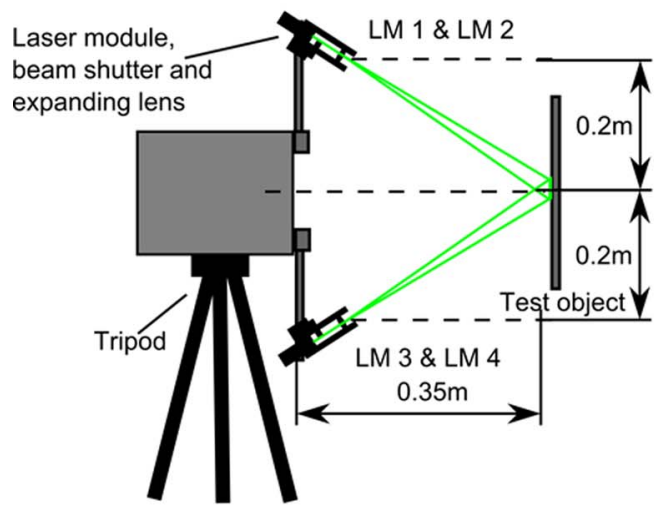

SIDE VIEW

\section{Experimental System}

\section{A. Experimental Arrangement}

The system used in this investigation is a multicomponent shearography system using time-division multiplexing of the measurement channels. Figure 3 shows a schematic and photograph of the experimental arrangement. This consisted of four diode pumped solid-state lasers (PHOTOP Suwtech Inc, Model DPGL-3020F, $532 \mathrm{~nm}, 20-30 \mathrm{~mW}$ output power) with mechanical shutters and beam expanding optics, mounted at the corners of a square using a crossshaped support structure. A shearing Michelson interferometer, camera lens, and a CCD camera records interferometric speckle images of the test object from the center of this arrangement. The shearing Michelson interferometer contains a beam splitter, a shearing mirror that can be adjusted to give different magnitudes and directions of applied shear, and a reference mirror that incorporates a piezoelectric transducer phase-stepping device. The camera is an area scan CCD camera (DALSA, Model CA-D4-0512A, $75 \mathrm{~Hz}$ frame rate, $512 \times 512$ pixels) and is used in conjunction with a frame grabber card (Bitflow Inc., Roadrunner, PCI frame grabber card). A personal computer, operating using software written in

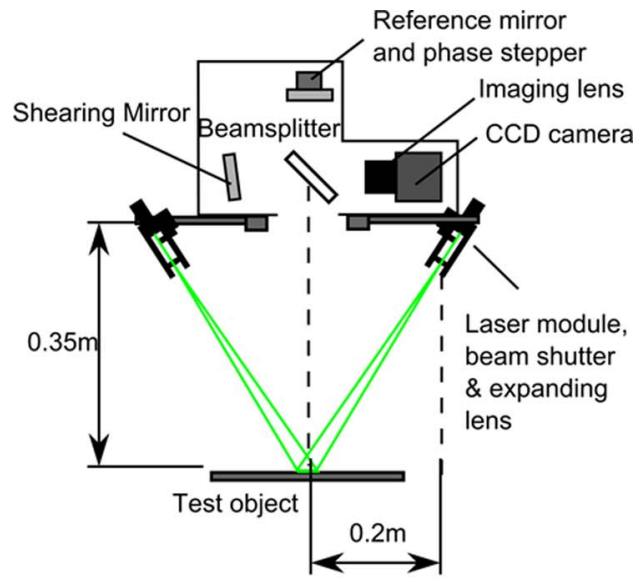

PLAN VIEW

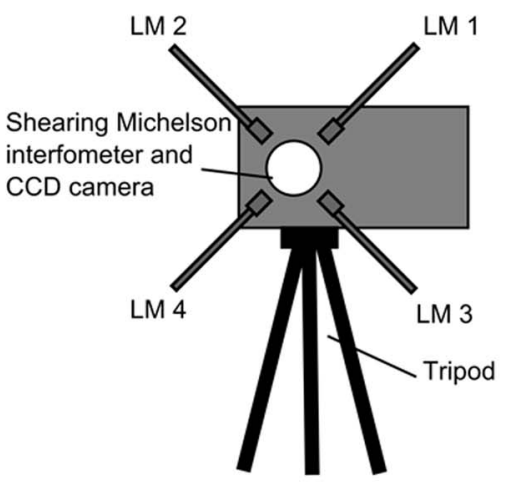

FRONT VIEW

Fig. 3. (Color online) Schematic and photograph of the multicomponent shearography system used in this investigation. LM, laser module, shutter and beam expanding optics; MSI, Michelson shearing interferometer; CCD, charge-coupled device camera. 
National Instruments LabVIEW, controls the switching of the lasers, the phase stepping of the reference mirror, and synchronized image capture by the camera.

\section{B. Experimental Data Collection and Processing}

Data are captured for each measurement channel sequentially. The object is illuminated by light from a single laser and a sequence of five images is captured, each with a relative $\pi / 2$ phase step induced by a voltage applied to the piezoelectric phase stepper. The phases are then calculated using a five step algorithm proposed by Hariharan et al. [15], which compensates well for errors introduced by miscalibration of the phase shifter:

$$
\phi=\tan ^{-1}\left\{\frac{2\left(I_{2}-I_{4}\right)}{2 I_{3}-I_{5}-I_{1}}\right\} .
$$

This process is then repeated for all four measurement channels and results in four phase images. The phase shifts $\left(\Delta \phi_{1}\right.$ to $\left.\Delta \phi_{4}\right)$ due to a deformation applied to the test object are then found by subtracting the phases measured before and after this deformation. The resulting phase shifts are wrapped between $-\pi$ and $\pi$ and need to be unwrapped prior to the calculation of the displacement gradients. Many different phase unwrapping algorithms exist, a discussion of which is beyond the scope of this paper; here, the "iso-phase unwrapping" algorithm proposed by Herráez et al. [6] was used. A sine-cosine filter was applied before this unwrapping to reduce high-frequency noise within the wrapped phase maps, as suggested by Aebischer and Waldner [16]. After the unwrapping, a $3 \times 3$ median filter was applied to remove single pixel discontinuities in the unwrapped phase that are artifacts resulting from the phase unwrapping algorithm. This resulted in four unwrapped phase images, one for each measurement channel, that are sensitive to different components of the displacement gradient. Finally, the orthogonal displacement gradients are calculated using three or four measurement channels, using Eqs. (7) and (10)-(12).

\section{Computer Model}

To allow for a quantitative investigation into the influence of additional channels on the transformation to orthogonal displacement gradient components, a computer model was used. The process used is described in the following subsections.

\section{A. Simulation of the Interferometric Speckle Images}

The interferometric speckle images were generated using a method similar to that used by Dolinko and Kaufmann [17], modified to apply to a phase-stepping shearography system. First, a theoretical displacement field $U=(u, v, w)$ was generated. From this and the defined magnitude of the shear, the theoretical displacement gradients, $\frac{\partial u}{\partial s}, \frac{\partial v}{\partial s}$, and $\frac{\partial w}{\partial x}$ were calculated. These are used later for comparison with the modeled results. A speckle field, $S_{1}(x, y)$, corresponding to the unsheared arm of the interferometer, is then generated according to

$$
S_{1}(x, y)=\exp \left\{i\left[\phi_{0}(x, y)+\phi_{U}(x, y)+\phi_{\text {step }}\right]\right\},
$$

where $\phi_{0}(x, y)$ is a randomly generated phase representing the phase distribution of the scattered light from the rough surface and $\phi_{\text {step }}$ is the additional phase shift applied during the phase stepping of this arm of the interferometer. The phase shift caused by the displacement of the surface, $\phi_{U}(x, y)$, can be calculated from the displacement field, $U(x, y)$, and the defined sensitivity vector, $\hat{k}(x, y)$, at each pixel and is given by

$$
\phi_{U}(x, y)=\frac{2 \pi}{\lambda}(\hat{k}(x, y) \cdot \hat{U}(x, y)) .
$$

A second speckle field, $S_{2}(x, y)$, corresponding to the shearing arm of the interferometer, is then generated in a similar way, but with the values of $\phi_{0}(x, y)$ shifted according to the desired shear magnitude and direction. This shifted phase distribution, $\phi_{0}^{\prime}(x, y)$, requires no additional phase step to be added, as this arm of the interferometer is not modified during phase stepping:

$$
S_{2}(x, y)=\exp \left\{i\left[\phi_{0}^{\prime}(x, y)+\phi_{U}(x, y)\right]\right\} .
$$

The resulting interferometric intensity distribution $I(x, y)$ is then calculated by combining the two speckle fields after the application of a circular low-pass filter in the Fourier domain [17]. The radius of this filter represents the circular aperture of the camera lens and determines the resulting speckle size:

$$
I(x, y)=\left|F^{-1}\left\{F\left[S_{1}(x, y)\right] H\right\}+F^{-1}\left\{F\left[S_{2}(x, y)\right] H\right\}\right|^{2},
$$

where $F$ and $F^{-1}$ denote the forward and inverse twodimensional Fourier transforms, respectively, and $H$ is the circular low-pass filter in Fourier space. By varying $H$, the speckle size of the simulation can be varied; here, a speckle size of $\sim 1$ pixel was used, representing a typical experimental setup.

To simulate the effect of experimental noise, random noise was added to the interferometric speckle images according to the properties of the camera used in the experimental system described in Section 3 . This included random contributions from the photon shot noise generated from a Poisson distribution, and camera dark current and readout noise generated from a normal distribution. Finally, the intensity values were truncated and limited to 8 bit resolution $(0$ 255 counts) to account for the analog-to-digital conversion process.

\section{B. Simulation of a Single Shearography Channel}

For a single shearography channel, two sets of interferometric speckle images are generated using the same initial phase distribution, $\phi_{0}(x, y)$. A reference set in which the phase shift due to displacement is 
zero and a signal set in which the phase shift due to the displacement are included. This is the same as for an experimental system where a reference phase must be measured before the surface deformation occurs. Each set consists of five images with a relative $\pi / 2$ phase step, $\phi_{\text {step }}$ applied to the unsheared arm of the interferometer. The reference and signal phases can then be calculated using the five step algorithm [15] given in Eq. (13).

The phase shift, $\Delta \phi(x, y)$, is found by subtracting the signal phase from the reference phase and applying a phase unwrapping algorithm to remove the $2 \pi$ discontinuities and obtain a continuous phase measurement. Before unwrapping, additional noise from a normal distribution was added to simulate noise sources other than CCD camera noise, such as vibrations, phase shifter inaccuracies, and thermally induced phase variations. The level of this noise was chosen to given comparable values, to the results from the experimental system described in Section 3 ; however, as this investigation concerns the propagation of errors, the actual level of error in each channel is unimportant and will depend on the system to be modeled.

The same processing as used in the experimental processing scheme described in Subsection 3.B was applied to simulated data. A sine-cosine smoothing filter [16] was applied before the "iso-phase unwrapping" algorithm [6] was used to unwrap the data, and afterward a $3 \times 3$ median filter was applied to remove single pixel discontinuities. The resulting phase shift is related to the displacement gradients as given in Eqs. (2) and (3).

\section{Simulation of Multichannel Shearography System}

To assess the performance of the methods, using three channels and more than three channels, to calculate the orthogonal components, multiple shearography channels were modeled for the same displacement field. This results in a simulated phase shift image for each channel of $\Delta \phi_{1}(x, y)$ to $\Delta \phi_{4}(x, y)$,


the orthogonal displacement gradients. This can either be done directly using any three of the channels or using all of the channels and the leastsquares method proposed in Section 2.

Typical phase shift images for four measurement channels are shown in Fig. 4 and the results of this transformation to the orthogonal displacement gradients are shown in Fig. 5, showing the theoretical displacement gradients, the simulated gradients calculated using three channels, and the simulated gradients calculated using four channels. The example results shown are for the shear applied in the $x$ direction and for a Gaussian out-of-plane displacement.

\section{Test Deformations}

Three different test deformations were used to assess the performance of the least-squares method using four measurement channels. Similar test deformations were used with the computer model (Section 4) and experimental system (Section 3) described above and the model settings, shown in Table 1 , where chosen to approximately match those used in the experimental system and allow for a comparison between the two.

\section{A. Zero Deformation}

The first test case is with zero deformation applied; this can be used as a simple estimate of the measurement error and for a comparison between the error level predicted by the model and those obtained experimentally. For the model, a displacement field with zeros for all components and all pixels was used. The test object was a flat aluminum plate, approximately $17 \mathrm{~cm} \times 17 \mathrm{~cm}$ and painted with reflective paint that was clamped around all four edges. This was illuminated sequentially by each laser and interferometric speckle images captured for each of the four measurement channels. This provided the reference phases; a second set of images captured shortly

Wrapped phases

$\Delta \phi_{1}$

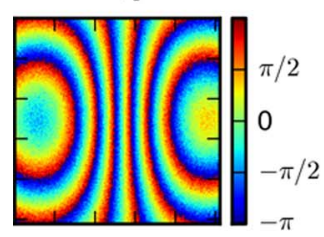

$\Delta \phi_{2}$

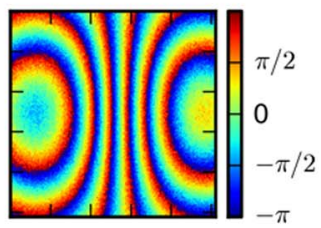

$\Delta \phi_{3}$

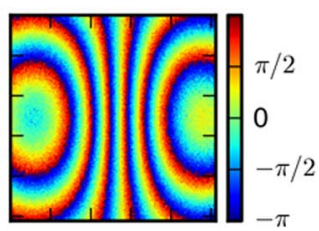

$\Delta \phi_{4}$



Unwrapped phases
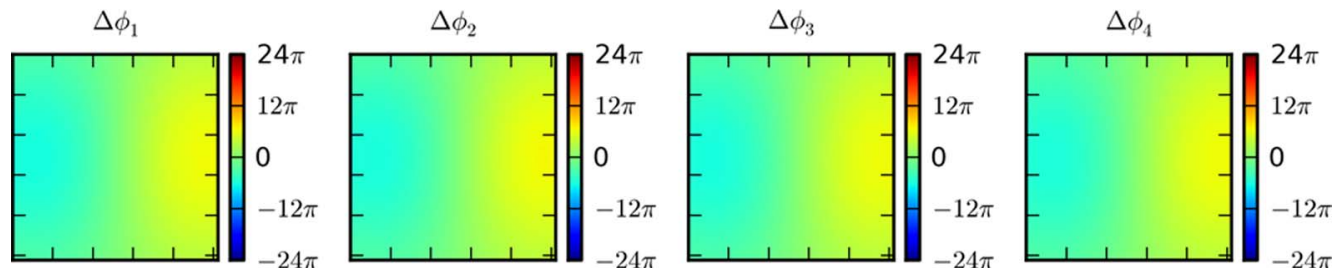

Fig. 4. (Color online) Simulated wrapped (top) and unwrapped (bottom) phase shifts for each of the four channels for a Gaussian out-ofplane displacement in the center of the field of view and including the noise sources. 

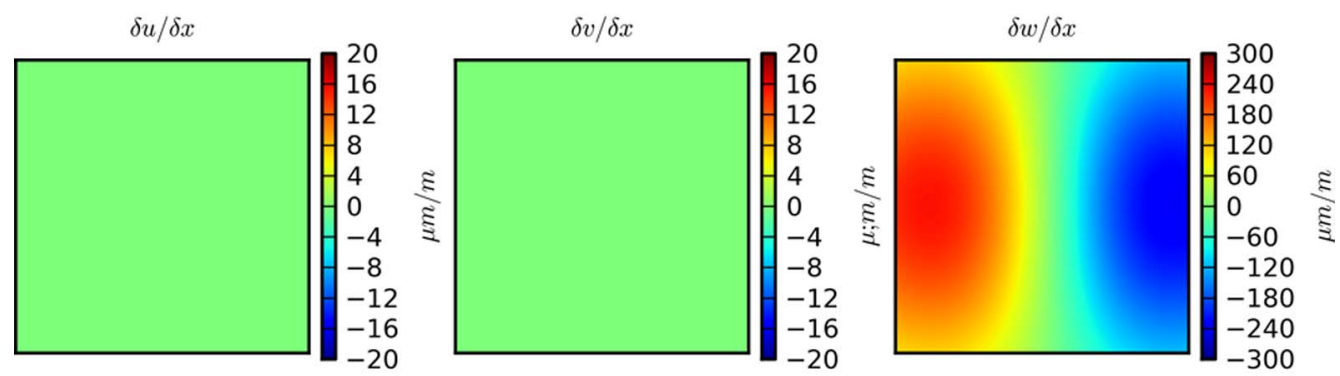

Modeled orthogonal displacement gradients calculated using three channels
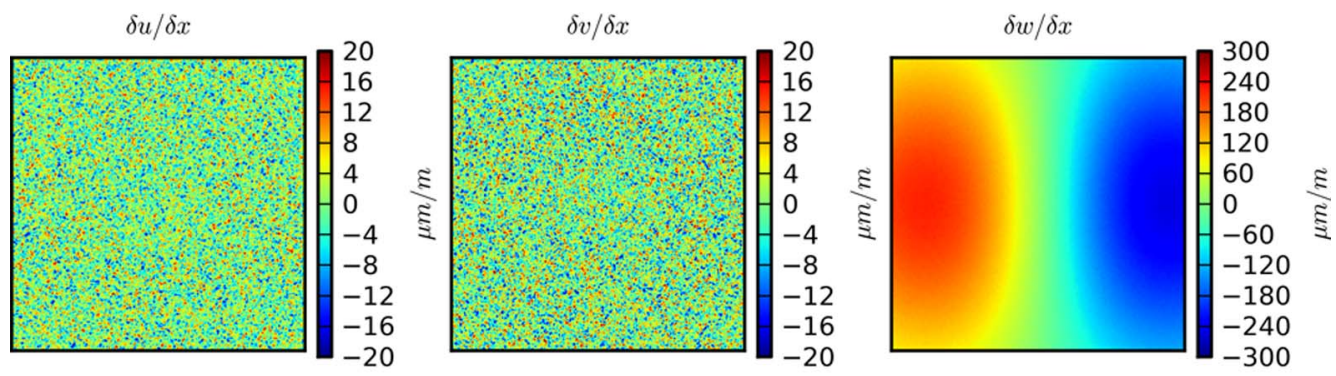

Modeled orthogonal displacement gradients calculated using four channels


Fig. 5. (Color online) Example results of the computer model for a Gaussian out-of-plane displacement in the center of the field of view. Top, theoretical displacement gradients; middle, displacement gradients calculated using three channels; and bottom, displacement gradients calculated using four channels.

Table 1. Settings Used in Multichannel Shearography Model, Chosen to Match Values Used in the Experimental System

\begin{tabular}{|c|c|}
\hline Model Property & Value \\
\hline Field of view & $62 \mathrm{~mm} \times 62 \mathrm{~mm}$ \\
\hline Shear magnitude & 33 pixels $/ 4 \mathrm{~mm}$ \\
\hline Speckle size ${ }^{a}$ & $\sim 1$ pixel \\
\hline Camera background noise mean ${ }^{b}$ & eight counts \\
\hline Camera background noise standard deviation ${ }^{b}$ & two counts \\
\hline Phase-stepping algorithm & five step [14] \\
\hline $\begin{array}{l}\text { Standard deviation of noise introduced } \\
\text { to wrapped phase shift }\end{array}$ & $\sim 0.17 \pi$ \\
\hline Filtering & $\begin{array}{l}\text { one pass of a } 3 \times 3 \text { sine-cosine smoothing filter }[16] \\
\text { before unwrapping } 1 \text { pass of } 3 \times 3 \text { median filter after unwrapping }\end{array}$ \\
\hline Phase unwrapping algorithm & "iso-phase unwrapping," Herráez et al. [] ] \\
\hline Observation vector, $\hat{o}$ & $(0.00,0.00,0.75) \mathrm{m}$ \\
\hline Channel 1 -illumination vector, $\hat{i}_{1}$ & $(-0.19,0.20,0.36) \mathrm{m}$ \\
\hline Channel 2 -illumination vector, $\hat{i}_{2}$ & $(0.20,0.18,0.35) \mathrm{m}$ \\
\hline Channel 3 -illumination vector, $\hat{i}_{3}$ & $(-0.22,-0.17,0.33) \mathrm{m}$ \\
\hline Channel 4-illumination vector, $\hat{i}_{4}$ & $(0.23,-0.19,0.33) \mathrm{m}$ \\
\hline
\end{tabular}

\footnotetext{
${ }^{a}$ Determined by radius of circular low-pass filter applied in the Fourier domain [17].

${ }^{b}$ Value determined from camera specifications and experimental characterization.

${ }^{c}$ Level determined by comparison with experimental data and represents noise sources other than camera noise.
} 

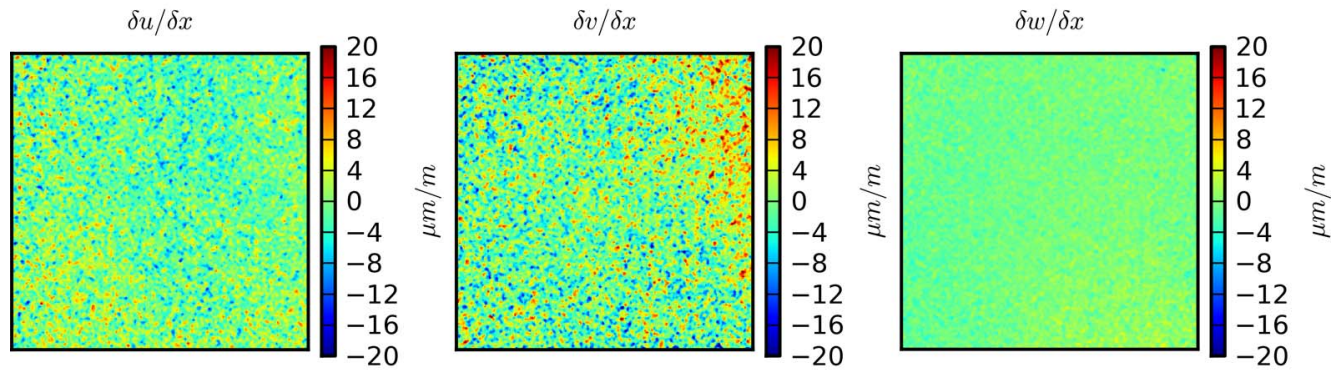

Experimental orthogonal displacement gradients calculated using four channels
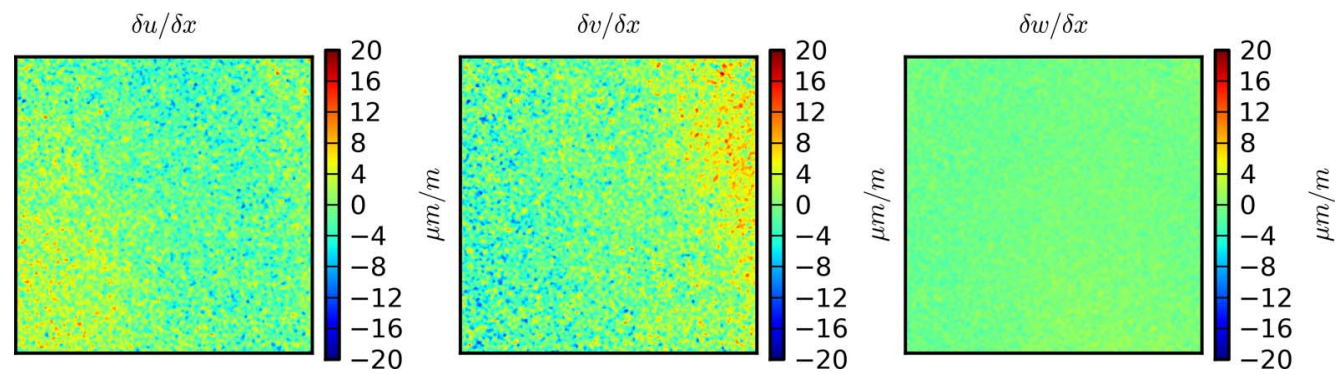

Fig. 6. (Color online) Calculated orthogonal displacement gradients for shear in the $x$ direction, calculated using the three (top row) and four (bottom row) channel methods for experimental results on a flat plate with zero deformation.

afterward was used for the signal phases. From these, the phase difference and the orthogonal displacement gradient components were calculated using three channels and using four channels. Figure $\underline{6}$ shows the calculated orthogonal displacement gradients for shear in the $x$ direction calculated using experimental results and Fig. $\underline{7}$ shows the same displace- ment gradients calculated using modeled results. Similar results can be obtained with shear in the $y$ direction. The standard deviation of the error present (the variation from the expected zero gradients) in these results is given in Table 2. As the values for the modeled results vary slightly between runs, due to variations in the speckle pattern and the

Modeled orthogonal displacement gradients calculated using three channels


Modeled orthogonal displacement gradients calculated using four channels
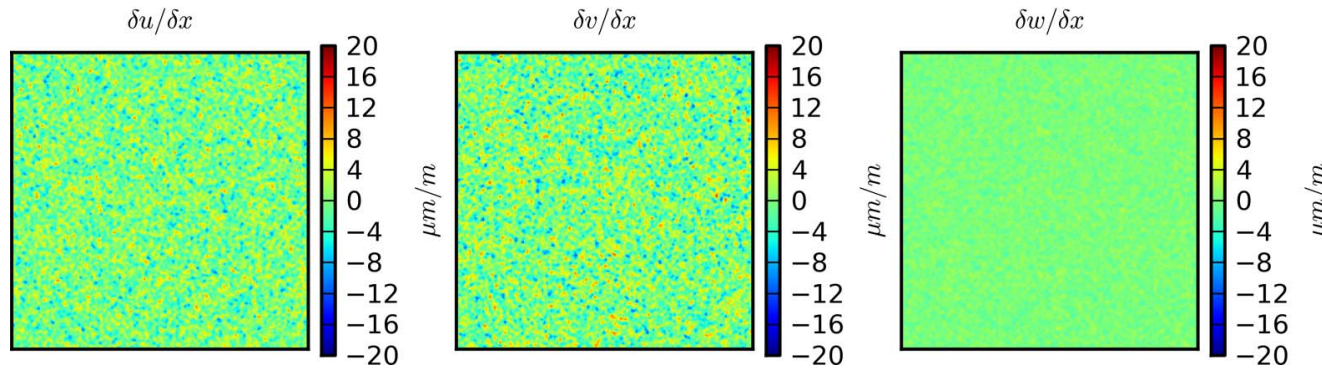

Fig. 7. (Color online) Calculated orthogonal displacement gradients for shear in the $x$ direction, calculated using the three (top row) and four (bottom row) channel methods for modeled data with zero deformation. 
Table 2. Results of the Zero Deformation Test for the Three-Channel and Four-Channel Methods

\begin{tabular}{|c|c|c|c|c|c|c|}
\hline & $\partial u / \partial x$ & $\partial v / \partial x$ & $\partial w / \partial x$ & $\partial u / \partial y$ & $\partial v / \partial y$ & $\partial w / \partial y$ \\
\hline Standard deviation in error $(\mu \mathrm{m} / \mathrm{m})-3$ channels & 3.71 & 5.20 & 1.24 & 3.32 & 3.26 & 1.02 \\
\hline Standard deviation in error $(\mu \mathrm{m} / \mathrm{m})-4$ channels & 3.03 & 3.59 & 0.81 & 2.22 & 2.44 & 0.70 \\
\hline$\%$ Reduction & $18.2 \%$ & $31.0 \%$ & $34.7 \%$ & $33.0 \%$ & $25.1 \%$ & $31.8 \%$ \\
\hline \multicolumn{7}{|l|}{ Modeled data (mean of 20 runs) } \\
\hline Standard deviation in error $(\mu \mathrm{m} / \mathrm{m})-3$ channels & 4.57 & 5.17 & 1.22 & 4.57 & 5.17 & 1.22 \\
\hline Standard deviation in error $(\mu \mathrm{m} / \mathrm{m})-4$ channels & 3.03 & 3.45 & 0.82 & 3.02 & 3.45 & 0.82 \\
\hline
\end{tabular}

performance of the phase unwrapping algorithm, the modeled results presented in the table are the mean average of 20 runs of the model.

It can be seen (Table 2) that the modeling results predict a significant reduction in the error propagation to the orthogonal displacement gradients of around $33 \%$, resulting in visibly less noisy results. The out-of-plane components $\frac{\partial w}{\partial x}$ and $\frac{\partial w}{\partial y}$ have lower noise levels due to the increased sensitivity of the system in this direction-this can be seen from examining the direction of the sensitivity vectors for the four channels. The experimental results show similar trends, although with slightly less improvement for some of the displacement gradients than predicted by the model. This appears to be due to the variation in illumination intensity across the field of view resulting from the Gaussian intensity profile of the illuminating lasers. This resulted in some variation in the error level across the images.

\section{B. Out-of-Plane Deformation}

The second test case was an out-of-plane deformation using the test object described in Subsection 5.A but deformed in the center using a micrometer screw to approximate a Gaussian deformation field. Figure 8 shows the orthogonal displacement gradients for shear in the $x$ direction calculated from experimental results. These show the expected form, with the displacement gradient predominantly in the out-ofplane direction; however, there is some displacement gradient present in the in-plane directions. From the results shown in Fig. 8, it can be seen that there is a visible reduction in the noise level for the orthogonal gradients computed using four channels compared to those calculated using only three. It is not possible to directly calculate the error in the experimental results as the true displacement is not known. However, the error present can be estimated by subtracting the local mean value from pixels in the image, leaving only the high-frequency noise. The local mean value was calculated by convolving the gradient images with $16 \times 16$ pixel averaging filter kernel. The standard deviation of the error and the percentage reduction when using four measurement channels are shown in Table 3 .

Experimental orthogonal displacement gradients calculated using three channels
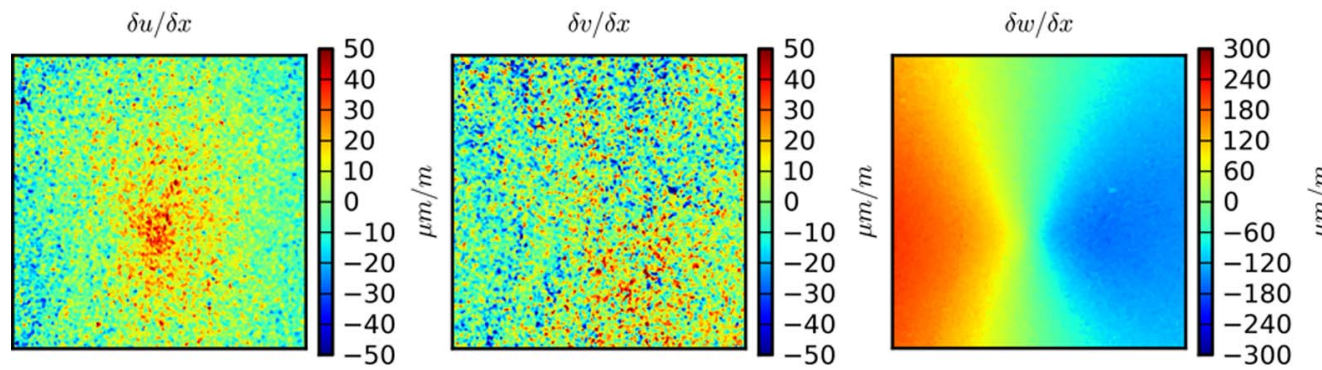

Experimental orthogonal displacement gradients calculated using four channels


Fig. 8. (Color online) Calculated orthogonal displacement gradients for shear in the $x$ direction, calculated using the three (top row) and four (bottom row) channel methods for experimental results on a flat plate with out-of-plane deformation. 
Table 3. Results of the Out-of-Plane Deformation Test for Experimental Data for the Three-Channel and Four-Channel Methods ${ }^{a}$

\begin{tabular}{|c|c|}
\hline & $\partial u / \partial x \partial v / \partial x \partial w / \partial x$ \\
\hline $\begin{array}{l}\text { Standard deviation in error }(\mu \mathrm{m} / \mathrm{m})-3 \\
\text { channels }\end{array}$ & $\begin{array}{lll}8.99 & 14.75 & 3.26\end{array}$ \\
\hline $\begin{array}{l}\text { Standard deviation in error }(\mu \mathrm{m} / \mathrm{m}) \_4 \\
\text { channels }\end{array}$ & $\begin{array}{lll}7.46 & 8.20 & 2.06\end{array}$ \\
\hline$\%$ Reduction & $17.1 \% 44.4 \% 36.8 \%$ \\
\hline
\end{tabular}

${ }^{a}$ These are the standard deviations of the estimated error calculated by subtracting the local mean.

This displacement was also modeled as a twodimensional Gaussian displacement, allowing the errors to be calculated directly. The out-of-plane displacement component is modeled by

$$
w(x, y)=w_{0} \exp \left\{-\left(\frac{x-x_{0}}{s_{x}}\right)^{2}-\left(\frac{y-y_{0}}{s_{y}}\right)^{2}\right\}
$$

where $w_{0}$ is the maximum out-of-plane displacement, $(x, y)$ are the position coordinates, $\left(x_{0}, y_{0}\right)$, is the center of the displacement, and $\left(s_{x}, s_{y}\right)$ is the scale of the displacement in the $x$ and $y$ directions, respectively. The in-plane displacement components, $u$ and $v$, are set to zero and the position coordinates are determined using the modeled camera resolution and field of view, shown along with other model properties in Table 1 . The value for $w_{0}$, the maximum out-of-plane displacement, was chosen to be $10 \mu \mathrm{m}$ to approximately match the magnitude of the displacement expected in the experimental test deformation. Figure 9 shows the displacement gradients calculated using the model for a $10 \mu \mathrm{m}$ maximum displacement in the out-of-plane direction.
Table 4. Results of the Out-of-Plane Deformation Test for Modeled Data (Mean of 20 Runs) for the Three-Channel and Four-Channel Methods

$\partial u / \partial x \partial v / \partial x \partial w / \partial x \partial u / \partial y \partial v / \partial y \partial w / \partial y$

$\begin{array}{lllllll}\text { Standard deviation in error } & 6.38 & 7.18 & 1.70 & 6.36 & 7.22 & 1.74\end{array}$

$(\mu \mathrm{m} / \mathrm{m})-3$ channels

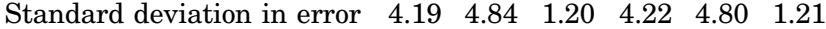

$(\mu \mathrm{m} / \mathrm{m}) \_4$ channels

$\%$ Reduction

$34.3 \% 32.5 \% 29.2 \% 33.7 \% 33.5 \% 30.7 \%$

To calculate the errors, the theoretical displacement gradients, $\frac{\partial u}{\partial s}, \frac{\partial v}{\partial s}$, and $\frac{\partial w}{\partial x}$, can then be calculated by using the magnitude of the camera shear and the theoretical displacement field with both the in-plane components, $\frac{\partial u}{\partial s}$ and $\frac{\partial v}{\partial s}$, being zero. These theoretical gradients are then subtracted from the modeled results, leaving only the error remaining; the mean standard deviation of these errors and the percentage reduction are shown in Table 4 . As before, the mean values of 20 runs of the model are given to remove variations due to speckle pattern variations and phase unwrapping errors. Although the experimental and modeled displacement fields are different, the modeling suggests that reductions in the standard deviation of the error of $25 \%$ to $30 \%$ should be achievable using four measurement channels and significant reductions are also seen in the estimated error for the experimental results.

\section{In-Plane Deformation}

As the previous test deformation had very little inplane components, a final test was performed to investigate the benefits of a fourth measurement channel for in-plane displacements. In this case, a Perspex plate with a notch cut-out was used, as shown in Fig. 10. A deformation was applied by tightening the
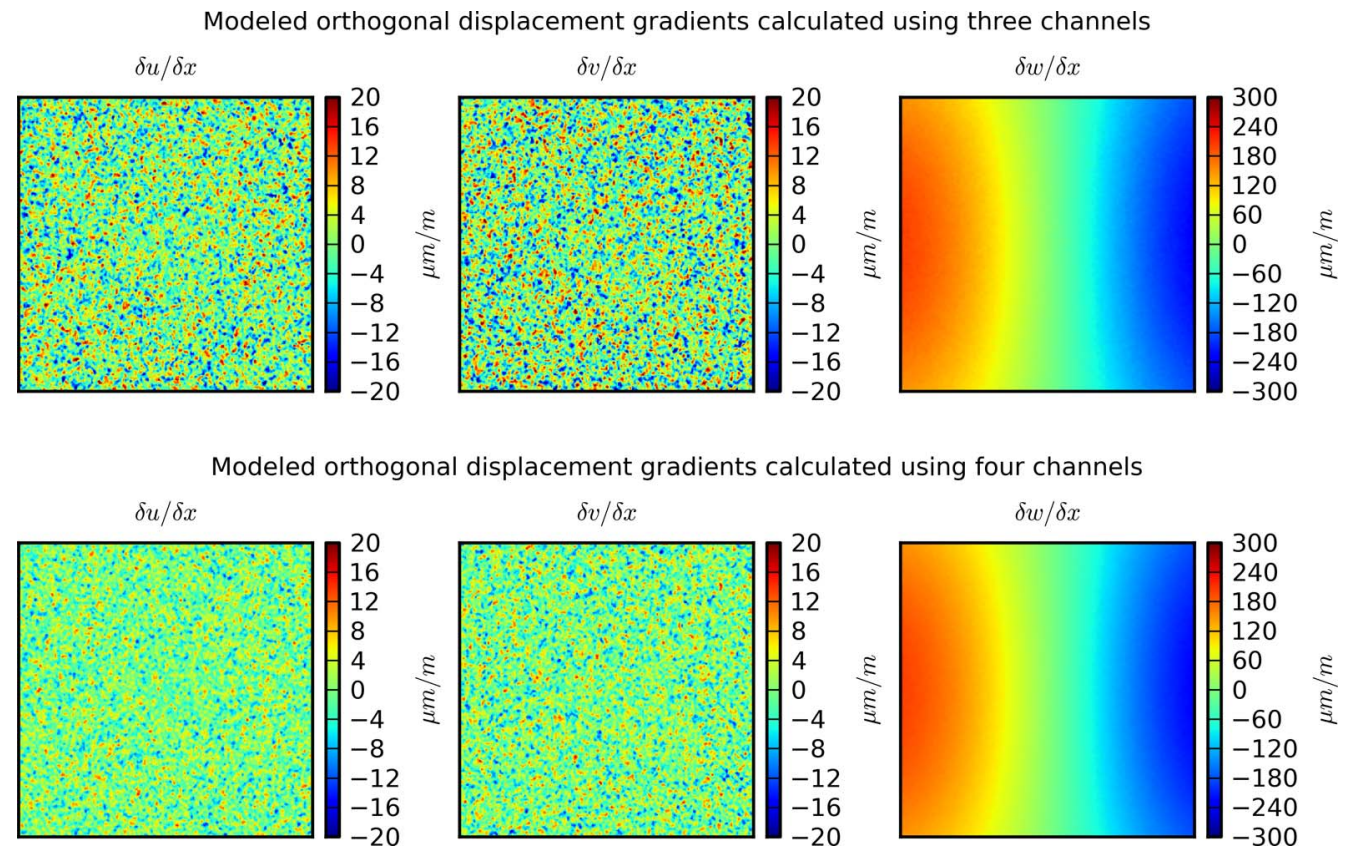

Fig. 9. (Color online) Calculated orthogonal displacement gradients for shear in the $x$ direction, calculated using the three (top row) and four (bottom row) channel methods for modeled data of a Gaussian out-of-plane deformation. 


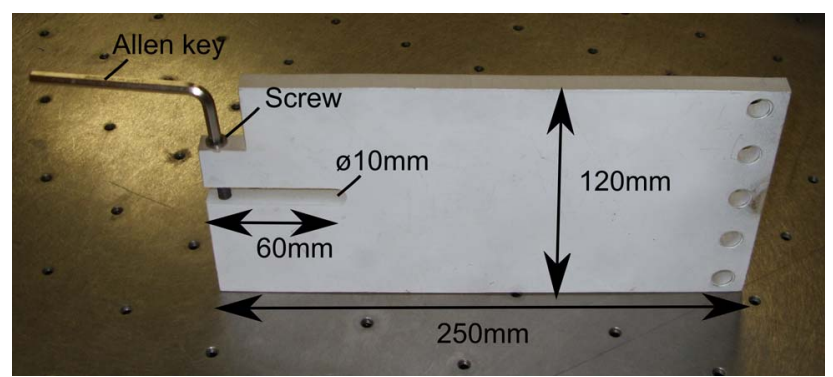

Fig. 10. (Color online) Photograph of the notch cut-out object used for the in-plane deformation test.

screw using the Allen key shown to close the notch. As this displacement gradient field would be complex to model, only experimental data are presented for this test case.

Figure 11 shows the calculated orthogonal displacement gradients for shear in the $y$ direction. The end of the notch is located approximately half-way up on the left-hand side of the images in the noisy region where there are no phase data. It can be seen that there is again some reduction in the noise present - this is especially visible in the $\frac{\partial u}{\partial y}$ components. As in the Subsection 5.B, the error could not be directly calculated as the displacement field is unknown, although an estimate of the error can be obtained by subtracting the local mean. The standard deviation of these estimated errors and the percentage reductions when using a fourth measurement channel are shown in Table 5 . These results again suggest that significant reductions in the error can be achieved by using a fourth measurement channel.

It should be noted that the addition of a fourth measurement channel will cause extra errors where the
Table 5. Results of the In-Plane Deformation Test for Experimental Data, for the Three-channel and Four-Channel Methods ${ }^{a}$

Experimental data

Standard deviation in error 27.1427 .9411 .8828 .0920 .919 .88

$(\mu \mathrm{m} / \mathrm{m})-3$ channels

Standard deviation in error $15.8421 .8411 .3921 .4917 .77 \quad 7.85$

$(\mu \mathrm{m} / \mathrm{m}) \_4$ channels

$\%$ Reduction

$41.6 \% 21.8 \% 4.14 \% 23.5 \% 15.0 \% 20.6 \%$

${ }^{a}$ These are the standard deviations of the estimated error calculated by subtracting the local mean.

additional channel has a region where the phase unwrapping has failed. This can be seen in Fig. 11 for the results calculated using four channels, where the fourth channel had a phase unwrapping issue in the bottom left portion of the field of view (shown by the rectangular overlay), resulting in this error propagating into all three calculated orthogonal components. This is also an issue when using three channels and, in previous work [9], data from a fourth channel has been used to replace one of the three channels used in the transformation. Although the addition of a fourth channel will increase the chances of a poorly unwrapped region occurring, this can be overcome by applying a weighting in the transformation, as given in Eq. (9). This could be based upon the results of the phase unwrapping for each channel and done on a pixel-by-pixel or regional basis with poorly unwrapped regions being given zero or low weighting - effectively calculating the gradients at these points using only three channels.

Experimental orthogonal displacement gradients calculated using three channels


Experimental orthogonal displacement gradients calculated using four channels
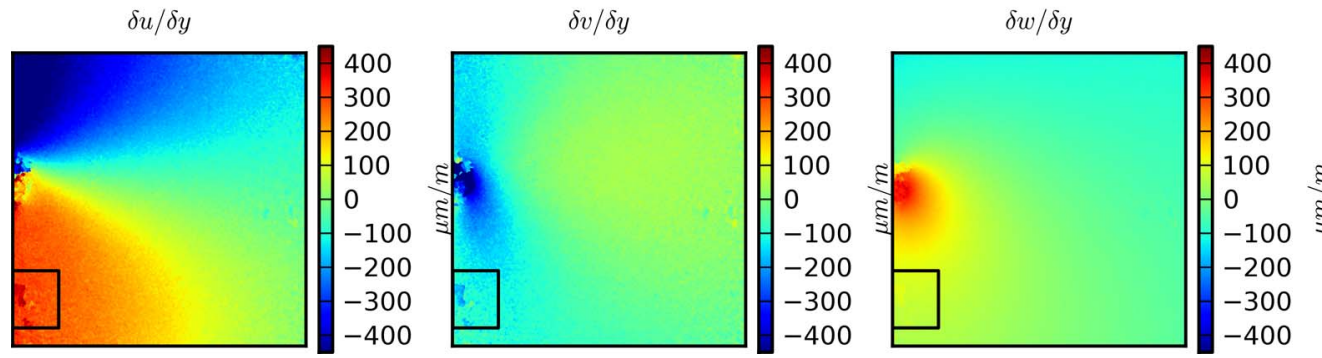

Fig. 11. (Color online) Orthogonal displacement gradients for shear in the $y$ direction calculated using the three (top row) and four (bottom row) channel methods from experimental results from the Perspex notch test object. 



Fig. 13. (Color online) Modeled standard deviations in the error and percentage reduction in comparison to a three channel system for $n$-channel configurations ( $n=3$ to 10). The results for all three orthogonal displacement gradients $(\cdot) \frac{\partial u}{\partial s},(\times) \frac{\partial v}{\partial s}$, and $(+) \frac{\partial w}{\partial s}$ are shown.

\section{More than Four Measurement Channels}

The least-squares method of calculating the orthogonal displacement gradients presented is not limited to four measurement channels. Although adding more than four channels to an experimental system may be complex, a system using scanning laser illumination or object rotation and sequential data capture could be envisaged. Therefore, the benefits of using more than four channels were investigated using the computer model. Multiple measurement channels were generated using multiple illumination directions and a single observation direction. The observation vector was set perpendicular to the surface at $z=0.75 \mathrm{~m}$ and the illumination sources where evenly distributed around a circle of radius $r=0.28 \mathrm{~m}$ in the $x-y$ plane, together with the observation position at $z=0.75 \mathrm{~m}$. Figure 12 shows the geometries modeled for $n=3,4,5$, and 6 channel systems; for systems with more channels, the illumination sources were similarly evenly distributed around the circle.

These geometries were then modeled using a zero displacement field as described in Subsection 5.A, and the orthogonal displacement gradients were calculated using all of the available measurement channels. The standard deviation in the error and the percentage reduction in error in comparison to a three

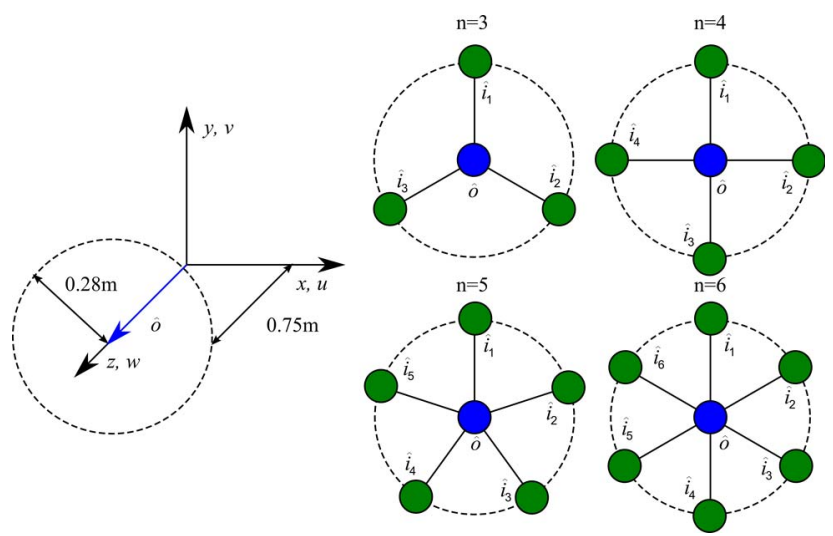

Fig. 12. (Color online) Multiple channel shearography system geometries used showing the arrangement of illumination sources and observation vector for $n$ channels ( $n=3$ to 6 ). channel system are shown plotted in Fig. 13. These plots show the results for the three orthogonal displacement gradients and configurations with $n=3$ to $n=10$ channels.

The first observation should be that the reduction in propagated error when a fourth measurement channel is added is less than in the previous sections, $\sim 14 \%$ compared to $\sim 33 \%$. This is due to the optimum configuration for three channels [9] being used here; i.e., with the three illumination positions evenly spaced around the circumference of a circle compared to the previous sections, where the illumination positions were separated by $90^{\circ}$. This nonoptimum configuration was chosen to facilitate the addition of a fourth channel to the experimental system.

The results show that the addition of extra measurement channels leads to reductions in the propagated errors, albeit with diminishing returns. The addition of a fifth channel leads to a reduction of $\sim 9 \%$, while adding a sixth gives a further reduction of only $6.6 \%$. All of the orthogonal displacement gradients, shown in Fig. 13 by different symbols, show similar reductions; however, the magnitude of the errors for the $\frac{\partial w}{\partial s}$ gradient are smaller than for the inplane gradients due to the increased sensitivity of the system to the out-of-plane component.

\section{Conclusions}

A method of calculating the orthogonal displacement gradients in a multicomponent shearography system using more than the minimum three channels required and a least-squares approach has been presented. The benefits of this approach were then studied using an experimental multicomponent shearography system using multiple illumination directions and single observation direction to provide four measurement channels, and a computer model of this system.

It was difficult to assess the performance of the experimental system for test deformations directly, because the actual displacement field was unknown. However, the results of a test where the displacement is known to be zero suggest that the 
standard deviation of the error in the calculated orthogonal components can be reduced by around $33 \%$ by the addition of a fourth measurement channel. This agrees well with the predictions of the computer model for the same zero displacement. Example measurements of objects subjected to outof-plane and in-plane surface displacement shows visible reductions in the noise. Modeled results for a Gaussian out-of-plane displacement suggest that the reductions in the standard deviation of the error are again around $33 \%$. Reductions in the error are also observed for other test deformations where the error is estimated by subtracting the local mean value of the displacement gradient to leave only high-frequency noise.

The primary disadvantages of using additional measurement channels is an increase in system complexity and increased errors when the phase unwrapping has difficulties. A fourth measurement channel is actually relatively inexpensive and straightforward to implement, either using a fourth illumination direction (as described here) or using imaging fiber bundles [11]. The addition of a fourth measurement channel will increase the chances of having regions where the phase unwrapping fails, which results in errors in the three orthogonal components, compared with using only three channels. However, this can be mitigated by assessing the performance of the unwrapping algorithm and applying a weighting scheme in the calculation. By applying a zero or low weighting to regions of the affected measurement channel, the orthogonal components would effectively be calculated using only three components and this could be done on either a pixel-by-pixel or regional basis.

The addition of further channels to a shearography system will lead to further reductions in the propagated errors, although with diminishing returns and, for practical systems, the added cost and complexity associated with this may outweigh the advantage of this reduction.

This work was supported by the Engineering and Physical Sciences Research Council (EPSRC), UK (GR/T09149 and EP/H019839).

\section{References}

1. J. Leendertz and J. Butters, "An image-shearing specklepattern interferometer for measuring bending moments," J. Phys. E 6, 1107-1110 (1973).
2. Y. T. Hung, "Shearography: a new optical method for strain measurement and nondestructive testing," Opt. Eng. 21, 391-395 (1982).

3. D. Francis, R. P. Tatam, and R. M. Groves, "Shearography technology and applications: a review," Meas. Sci. Technol. 21, 102001 (2010).

4. M. Kalms and W. Osten, "Mobile shearography system for the inspection of aircraft and automotive components," Opt. Eng. 42, 1188-1196 (2003).

5. K. Creath, "Phase-shifting speckle interferometry," Appl. Opt. 24, 3053-3058 (1985).

6. M. A. Herráez, M. A. Gdeisat, D. R. Burton, and M. J. Lalor, "Robust, fast, and effective two-dimensional automatic phase unwrapping algorithm based on image decomposition," Appl. Opt. 41, 7445-7455 (2002).

7. S. Waldner and S. Brem, "Compact shearography system for the measurement of 3D deformation," Proc. SPIE $\mathbf{3 7 4 5}$, 141-148 (1999).

8. R. Kästle, E. Hack, and U. Sennhauser, "Multiwavelength shearography for quantitative measurements of twodimensional strain distributions," Appl. Opt. 38, 96-100 (1999).

9. R. M. Groves, S. W. James, and R. P. Tatam, "Multi-component shearography employing four measurement channels," Proc. SPIE, 4933 135-140 (2003).

10. D. Francis, S. W. James, and R. P. Tatam, "Surface strain measurement using multi-component shearography with coherent fibre-optic imaging bundles," Meas. Sci. Technol. 18, 3583-3591 (2007).

11. D. Francis, S. W. James, and R. P. Tatam, "Surface strain measurement of rotating objects using pulsed laser shearography with coherent fibre-optic imaging bundles," Meas. Sci. Technol. 19, 105301 (2008).

12. D. S. Nobes, H. D. Ford, and R. Tatam, "Instantaneous, threecomponent planar Doppler velocimetry using imaging fibre bundles," Exp. Fluids 36, 3-10 (2004).

13. T. O. Charrett, D. S. Nobes, and R. Tatam, "Investigation into the selection of viewing configurations for three-component planar Doppler velocimetry measurements," Appl. Opt. 46, 4102-4116 (2007).

14. K. Arbenz and A. Wohlhauser, Advanced Mathematics For Practicing Engineers (Artech House, 1986).

15. P. Hariharan, B. Oreb, and T. Eiju, "Digital phaseshifting interferometry: a simple error-compensating phase calculation algorithm," Appl. Opt. 26, 2504-2506 (1987).

16. H. Aebischer and S. Waldner, "A simple and effective method for filtering speckle-interferometric phase fringe patterns," Opt. Commun. 162, 205-210 (1999).

17. A. Dolinko and G. H. Kaufmann, "A least-squares method to cancel rigid body displacements in a hole drilling and DSPI system for measuring residual stresses," Opt. Lasers Eng. 44, 1336-1347 (2006). 